\title{
DETC99/DAC-8561
}

\section{AUTOMATED METHODS IN COMPUTER AIDED DESIGN}

\author{
K Abdel-Malek \\ Department of Mechanical Engineering, \\ The University of lowa, lowa City, IA 52242 \\ Tel. (319) 335-5676 \\ amalek@engineering.uiowa.edu \\ J-Y Wang \\ Structural Dynamics Research Corporation \\ (SDRC), Research and Development Department, \\ Milford, $\mathrm{OH}$,
}

\author{
$\mathrm{H}-\mathrm{L}$ Zou \\ Structural Dynamics Research Corporation \\ (SDRC), Research and Development \\ Department, Milford, $\mathrm{OH}$ \\ denise.zou@heimdall.sdrc.com \\ S Othman \\ US National Advanced Driving Simulator \\ (NADS), Oakdale, IA, USA \\ Othman@nads-sc.uiowa.edu
}

\begin{abstract}
A numerical variational method is herein introduced for the automatic redesign of mechanical parts subject to a design change. This method is demonstrated to the design of mechanical parts in the context of concurrent engineering of 3D parts. Relations between dimensions in a mechanical part as dictated by a designer are modeled using mechanisms and their kinematic relations. Variational cut-joint constraints are introduced, kinematic joints in the formulation are combined, and a Jacobian is determined. Constraint violations are then compensated to compute an assembled mechanism, hence redesigning the part. It is shown that this formulation is broadly applicable to 2D and 3D models. The method and algorithm are illustrated through examples.
\end{abstract}

\section{INTRODUCTION}

Commercial computer aided design and manufacturing (CAD/CAM) computer code have provided versatile venues for end-users to automate the design process. Despite recent advances, the sophistication of these formulations remains at an early stage of development. Learning and creating new methods and methodologies for computers to provide more aid to the designer is a goal worth hundreds of millions of dollars to the world economy. The goal is to automate the product development process within the mechanical CAD/CAM environment.
Computer code that implements the iterative concept of design and that automatically alters designs is reported by many researchers [1-3]. This type of software facilitates constraintbased modeling and in many cases provides inferencing via constraint propagation. A design change in the geometry of a part propagates through the complete design. Other efforts at $\mathrm{CAD} / \mathrm{CAM}$ automation include reasoning schemes that use topological relationships between features [4]. More recent efforts to automate the mechanical design process in the manufacturing field were demonstrated by Abdel-Malek and Maropis [5].

Derivation of basic constraints in the context of automatic CAD was first introduced by Zou, et al. [6]. It is based on a computational method for studying the kinematics of mechanical systems as first introduced in by Haug [7]. Basic constraints were first developed for the study of variational propagations in mechanism assembly design [8]. The extension of this work to the design of 3D mechanical parts, the combination of joints in the analysis, and the implementation of the algorithm in a concurrent engineering environment are herein presented.

General conventions used throughout the development of the method are first introduced in Section 2. The formulation will then be used to model the system using a spanning tree and a cut-joint constraint. A set of equations will be developed while maintaining vector quantities representing link length and 
orientation as variables, namely the Euler angles and the bodyposition vector (Section 3). These equations will then be linearized but having a non-square Jacobian. The MoorePenrose pseudo inverse will be used to compute an assembled configuration, hence automatically redesigning the mechanism subject to the specified change (Section 4). The new mechanism will then be converted back to a mechanical part, hence providing a new design. Two examples will be illustrated in the context of computer-aided engineering. The ultimate goal of this research is to develop a concurrent engineering environment that automates the product development process.

\section{CONVENTIONS}

The following conventions will be used throughout this formulation.

(1) Mechanical parts will be represented by a number of joints and links. The goal is to relate geometric dimensions by imposing constraints inherent in the mathematics of kinematic pairs. For example, consider the mechanical part shown in Fig. 1a. By choosing the appropriate types of joints, the designer allows for the distortion of the part in a particular manner. For example, two sliding joints $\left(\mathrm{J}_{2}\right.$ and $\left.\mathrm{J}_{3}\right)$, a revolute joint $\mathrm{J}_{4}$, and spherical joints $\left(\mathbf{J}_{1}, \mathbf{J}_{5}, \mathbf{J}_{6}, \mathbf{J}_{7}\right.$, and $\left.\mathbf{J}_{8}\right)$ are introduced as shown in Fig. 1b. The kinematic model of the part is shown in Fig. 1c where the proposed links are numbered 0 through 6 and the mechanical part has been modeled as a mechanism.
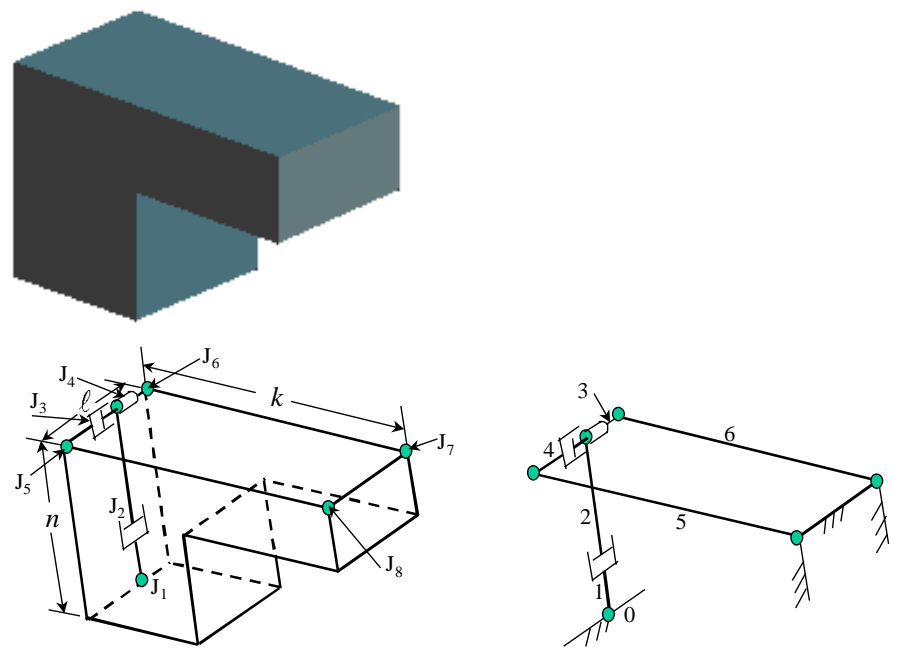

Fig. 1 (a) Mechanical part (b) Modeling the part as joints and links (c) The mechanism representation of the part

(2) A mechanism will be transformed into a graph. Each body is defined as a node and a kinematic joint is defined as an edge. For example, the scissors jack shown in Fig. 2a will be transformed into a graph representation as shown in Fig. 2b.

(3) A Graph representation will be cut to produce a tree structure. For example, the graph of Fig. $2 b$ is cut as shown in Fig. 2c to obtain a tree.

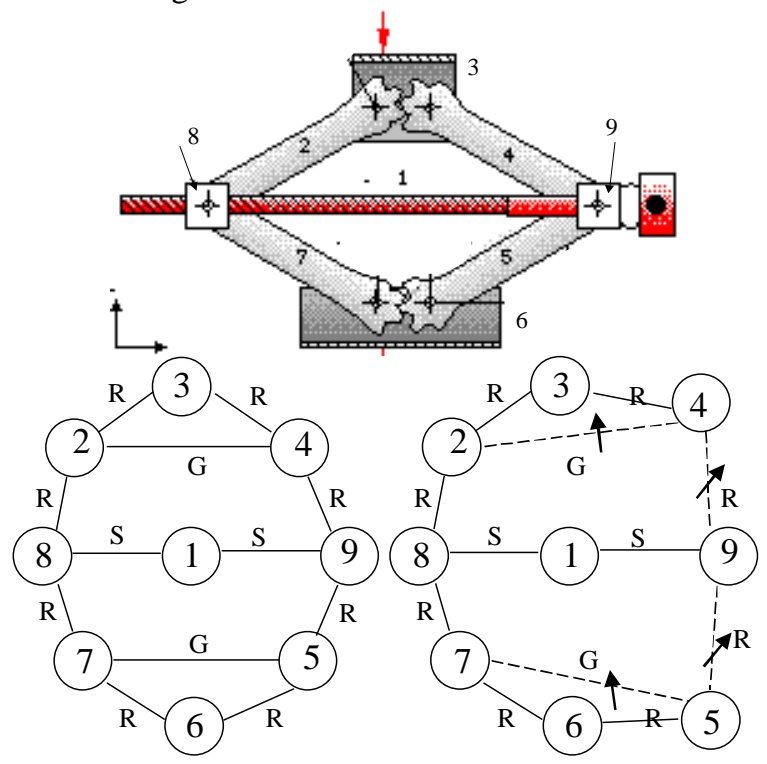

Fig. 2 (a) Scissors jack (b) A graph representation (c) Cutting joints to form a tree

(4) Vector notation used throughout this formulation is introduced in this section. In order to facilitate the forthcoming discussion, Fig. 3 illustrates the nomenclature used throughout.

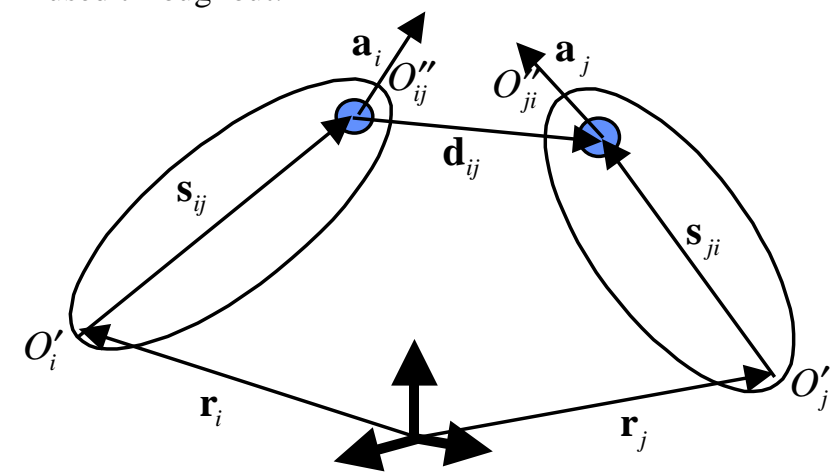

Fig. 3 Identification of coordinate systems

Define the vectors $\mathbf{a}_{i}$ and $\mathbf{a}_{j}$ as nonzero vectors fixed in the bodies $i$ and $j$, respectively. Define the vectors $\mathbf{r}_{i}$ and $\mathbf{r}_{j}$ as the global position vectors extending from the origin of the global reference frame to the origin of the body reference frame. Define the vectors $\mathbf{s}_{i j}$ and $\mathbf{s}_{j i}$ as the joint-attachment vectors in the body reference frame, where these vectors will be maintained as variables such that their variations will be automatically computed. 


\section{PROBLEM FORMULATION}

In this section, and throughout this derivation, the position vector embedded in each link and its orientation matrix are maintained as variables. The goal is to obtain linearized equation characterizing the assembly (i.e., mechanical part).

\subsection{A Review of Constraints (Zou, et al. 1996)}

A spherical joint is a type of constraint that requires a pair of points on two bodies to coincide. A necessary and sufficient condition for $O_{i j}^{\prime \prime}$ and $O_{j i}^{\prime \prime}$, to coincide is that $\mathbf{d}_{i j}=\mathbf{0}$; i.e.,

$$
\Phi^{s}\left(O_{i j}^{\prime \prime}, O_{j i}^{\prime \prime}\right)=\mathbf{r}_{j}+\mathbf{s}_{j i}-\mathbf{r}_{i}-\mathbf{s}_{i j}=\mathbf{0}
$$

The vectors $\mathbf{S}_{i j}$ and $\mathbf{S}_{j i}$ can be written as $\mathbf{S}_{i j}=\mathbf{A}_{i} \mathbf{S}_{i j} \quad$ and $\mathbf{S}_{j i}=\mathbf{A}_{j} \mathbf{S}_{j i}^{\prime}$. The variation of the joint position vectors can be written as

$$
\delta \mathbf{s}_{i j}=-\widetilde{\mathbf{s}}_{i j} \delta \pi_{i}+\mathbf{A}_{i} \delta \mathbf{s}_{i j}^{\prime}
$$

Taking the variation of Eq. (1) and substituting for $\delta \mathbf{S}_{i j}$ and $\delta \mathbf{s}_{j i}$ yields

$\delta \Phi^{s}=\delta \mathbf{r}_{j}-\delta \mathbf{r}_{i}-\widetilde{\mathbf{s}}_{j i} \delta \pi_{j}+\mathbf{A}_{j} \delta \mathbf{s}_{j i}^{\prime}+\widetilde{\mathbf{s}}_{i j} \delta \pi_{i}-\mathbf{A}_{i} \delta \mathbf{s}_{j i}^{\prime}$

where the matrices $\mathbf{A}_{i}$ and $\mathbf{A}_{j}$ represent direction cosine matrices from link reference frame $x^{\prime} y^{\prime} z^{\prime}$ to the global reference frame $x y z$; the symbol $(\sim)$ called tilde, is used to denote a skew-symmetric matrix generated by its corresponding vector (e.g., $\widetilde{\mathbf{a}}$ is the skew symmetric matrix generated from the vector $\mathbf{a}$ ); the matrix $\pi$ can be generated from the elements of $\pi=\delta \mathbf{A} \mathbf{A}^{T}$.

A distance constraint requires a specified distance between a pair of points. A necessary condition that the distance between $O_{i j}^{\prime \prime}$ and $O_{j i}^{\prime \prime}$ (Fig. 1), be equal to $\ell \neq 0$ is formulated as

$$
\Phi^{D}\left(O_{i j}^{\prime \prime}, O_{i j}^{\prime \prime}, \ell\right)=\mathbf{d}_{i j}^{T} \mathbf{d}_{i j}-\ell^{2}=\mathbf{0}
$$

and its variation is

$$
\delta \Phi^{D}=2 \mathbf{d}_{i j}^{T} \delta \mathbf{d}_{i j}=2 \mathbf{d}_{i j}^{T}\left[\delta \mathbf{r}_{j}-\delta \mathbf{r}_{i}-\widetilde{\mathbf{s}}_{j i} \delta \pi_{j}+\mathbf{A}_{j} \delta \mathbf{s}_{j i}^{\prime}+\widetilde{\mathbf{s}}_{i j} \delta \pi_{i}-\mathbf{A}_{i} \delta \mathbf{s}_{i j}^{\prime}\right]
$$

where the variation of $\mathbf{d}_{i j}$ is

$$
\delta \mathbf{d}_{i j}=\delta \mathbf{r}_{j}-\delta \mathbf{r}_{i}-\widetilde{\mathbf{s}}_{j i} \delta \pi_{j}+\mathbf{A}_{j} \delta \mathbf{s}_{j i}^{\prime}+\widetilde{\mathbf{s}}_{i j} \delta \pi_{i}-\mathbf{A}_{i} \delta \mathbf{s}_{i j}^{\prime}
$$

Combination of joints is performed in the analysis to simplify computations. Consider, for example, the translational and distance constraints: If the two joints are connected to each other in one branch, then they can be combined in the analysis and will be denoted by $T-D$.

The constraint equation that characterizes this joint is

$$
\Phi^{T-D}=\mathbf{I}_{i j}^{T} \mathbf{I}_{i j}-\ell_{T-D}^{2}
$$

where $\mathbf{I}_{i j}=\mathbf{d}_{i j}-\mathbf{h}_{i j} q_{3}$ and $\mathbf{h}_{i j}=\mathbf{A}_{i} \mathbf{C}_{i j}\left[\begin{array}{lll}0 & 0 & 1\end{array}\right]^{T}$.

Substituting Eq. (6) into (5) and using the identities derived, the variation of $\Phi^{T-D}$ is

$$
\begin{gathered}
\delta \boldsymbol{\Phi}^{T-D}=2 \mathbf{I}_{i j}^{T}\left[\delta \mathbf{r}_{j}+\mathbf{A}_{j} \delta \mathbf{s}_{j i}^{\prime}-\widetilde{\mathbf{s}}_{j i} \delta \boldsymbol{\pi}_{j}-\delta \mathbf{r}_{i}-\mathbf{A}_{i} \delta \mathbf{s}^{\prime}+\right. \\
\left.\left(\widetilde{\mathbf{s}}_{i j}+\widetilde{\mathbf{h}}_{i j} q_{3}\right) \delta \boldsymbol{\pi}_{i}+\widetilde{\mathbf{h}}_{i j} q_{3} \delta \xi_{i j}-\mathbf{h}_{i j} \delta q_{3}\right]-2 \ell_{T-D} \delta \ell_{T-D}
\end{gathered}
$$

\subsection{Jacobian Matrices}

The Jacobian matrix of a composite $T-D$ joint in Cartesian space can be written as

$$
\Phi_{\hat{\mathbf{z}}_{j}}^{T-D}=\left[\begin{array}{ll}
\Phi_{\mathbf{r}_{j}} & \Phi_{\pi_{j}}
\end{array}\right]\left[\begin{array}{cc}
\mathbf{I} & -\widetilde{\mathbf{r}}_{j} \\
\mathbf{0} & \mathbf{I}
\end{array}\right]
$$

where the symbol $\Phi_{\hat{\mathbf{z}}_{j}}$ denotes the Jacobian matrix of the constraint function $\Phi$ with respect to the coordinate space of $\hat{\mathbf{z}}$ (i.e., $\left[\partial \Phi_{i} / \partial \hat{\mathbf{z}}_{j}\right]$ ), and can be written as

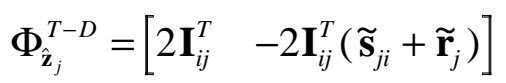

The Jacobian matrix is

$$
\Phi_{q_{3}}^{T-D}=-2 \mathbf{I}_{i j}^{T} \mathbf{h}_{i j}
$$

\subsection{Velocity Transformation Matrices of Combined Joints}

A Spherical-Translational (denoted by $S-T$ ) joint has four relative degrees of freedom $\mathbf{q}_{S-T}=\left[\begin{array}{ll}\mathbf{q}_{1} & q_{2}\end{array}\right]^{T}$, three for the spherical joint and one for the translational joint. Three relative rotational coordinates of the spherical joint can be expressed using Euler parameters. The Euler parameters are denoted by $e_{0}, e_{1}, e_{2}$, and $e_{3}$ such that the Euler vector is defined by

$\mathbf{p}_{1}=\left[\begin{array}{llll}\mathrm{e}_{0} & \mathrm{e}_{1} & \mathrm{e}_{2} & \mathrm{e}_{3}\end{array}\right]^{T}$. Since a translational joint does not have a rotational degree of freedom, the transformation matrix $\mathbf{A}_{i j}^{\prime \prime}$ of the $S-T$ joint is simply the same as that of a spherical joint, thus

$$
\mathbf{A}_{i j}^{\prime \prime}=\mathbf{A}_{s p h}^{\prime \prime}=\mathbf{E}_{j} \mathbf{G}_{j}^{T}
$$

where $\mathbf{E}_{j}$ and $\mathbf{G}_{j}$ are the Euler parameters semi-rotation matrices defined by Haug [7] as

$$
\mathbf{E}_{j}=\left[-\mathbf{e}_{j}, \tilde{\mathbf{e}}_{j}+\mathrm{e}_{j 0} \mathbf{I}\right]=\left[\begin{array}{cccc}
-\mathrm{e}_{1} & \mathrm{e}_{0} & -\mathrm{e}_{3} & \mathrm{e}_{2} \\
-\mathrm{e}_{2} & \mathrm{e}_{3} & \mathrm{e}_{0} & -\mathrm{e}_{l} \\
-\mathrm{e}_{3} & -\mathrm{e}_{2} & \mathrm{e}_{1} & \mathrm{e}_{0}
\end{array}\right]
$$


and

$$
\mathbf{G}_{j}=\left[-\mathbf{e}_{j},-\tilde{\mathbf{e}}_{j}+\mathrm{e}_{j 0} \mathbf{I}\right]=\left[\begin{array}{cccc}
-\mathrm{e}_{1} & \mathrm{e}_{0} & \mathrm{e}_{3} & -\mathrm{e}_{2} \\
-\mathrm{e}_{2} & -\mathrm{e}_{3} & \mathrm{e}_{0} & \mathrm{e}_{1} \\
-\mathrm{e}_{3} & \mathrm{e}_{2} & -\mathrm{e}_{1} & \mathrm{e}_{0}
\end{array}\right]
$$

and the transformation matrix $\mathbf{C}_{i j}$ can be represented as $\mathbf{C}_{i j}=\mathbf{E}_{j} \mathbf{G}_{j}^{T}$. The velocity transformation matrix $\mathbf{B}_{j}$ can be expressed as

$$
\mathbf{B}_{j}^{S-T}=\left[\begin{array}{ll}
\mathbf{B}_{s p h} & \mathbf{B}_{\text {tran }}
\end{array}\right]_{6 \times 5}
$$

where $\mathbf{B}_{s p h}$ is the velocity transformation for the spherical joint

$\mathbf{B}_{s p h}=\left[\begin{array}{ll}\widetilde{\mathbf{r}}_{j} & \mathbf{I}\end{array}\right]^{T} \mathbf{A}_{i} \mathbf{C}_{i j} 2 \mathbf{E}_{j}=\left[\begin{array}{ll}\widetilde{\mathbf{r}}_{j} & \mathbf{I}\end{array}\right]^{T} \mathbf{A}_{1} \mathbf{C}_{12 S-T} 2 \mathbf{E}_{j_{6 \times 4}}$

and $\mathbf{B}_{\text {tran }}$ is the velocity transformation for the translational joint $\mathbf{B}_{\text {tran }}=\left[\begin{array}{ll}\mathbf{g}_{i j} & \mathbf{0}\end{array}\right]^{T}$. Velocity transformation matrices are used to represent the variation of one body with respect to another. Assume bodies $i$ and $j$ are connected by two joints; after joint 1 is cut, body $i$ is the inboard body of body $j$.

In order to obtain a representation of one vector in terms of another in state vector form, and since the variation $\delta \mathbf{r}_{j}=\delta \mathbf{r}_{i}+\delta \mathbf{s}_{i j}+\delta \mathbf{d}_{i j}$, then substitute for $\delta \mathbf{d}_{i j}$ and $\delta \mathbf{s}_{i j}$ and use the relationship $\tilde{\mathbf{a}} \mathbf{b}=-\tilde{\mathbf{b}} \mathbf{a}$ yields

$$
\delta \mathbf{r}_{j}=\delta \mathbf{r}_{i}+\delta \tilde{\pi}_{i} \mathbf{s}_{i j}+\mathbf{A}_{i} \delta \mathbf{s}_{i j}^{\prime}+\delta \tilde{\pi}_{i} \mathbf{d}_{i j}-\widetilde{\mathbf{d}}_{i j} \delta \xi_{i j}^{\prime}+\frac{\delta \mathbf{d}_{i j}}{\delta \mathbf{q}_{j}} \delta \mathbf{q}_{j}
$$

Collecting terms and simplifying yields

$$
\delta \mathbf{r}_{j}=\delta \mathbf{r}_{i}+\delta \tilde{\pi}_{i}\left(\mathbf{r}_{j}-\mathbf{r}_{i}\right)+\mathbf{A}_{i} \delta \mathbf{s}_{i j}^{\prime}-\widetilde{\mathbf{d}}_{i j} \delta \xi_{i j}^{\prime}+\frac{\delta \mathbf{d}_{i j}}{\delta \mathbf{q}_{j}} \delta \mathbf{q}_{j}
$$

Adding a common term $\left(\tilde{\mathbf{r}}_{j} \delta \pi_{j}\right)$ to both sides, and using the derived identities yields

$$
\begin{aligned}
\delta \mathbf{r}_{j}+ & \widetilde{\mathbf{r}}_{j} \delta \pi_{j}=\delta \mathbf{r}_{i}+\widetilde{\mathbf{r}}_{i} \delta \pi_{i}+\mathbf{A}_{i} \delta \mathbf{s}_{i j i j}^{\prime} \\
& -\widetilde{\mathbf{d}}_{i j} \delta \xi_{i j}^{\prime}+\left(\frac{\delta \mathbf{d}_{i j}}{\delta \mathbf{q}_{j}}+\widetilde{\mathbf{r}}_{j} \mathbf{H}_{j}\right) \delta \mathbf{q}_{j}+\widetilde{\mathbf{r}}_{j} \delta \xi^{\prime}
\end{aligned}
$$

where $\mathbf{H}_{j}\left(\mathbf{A}_{i}, \mathbf{q}_{j}\right)$ is a transformation matrix that depends on the orientation of body $i$ and on the relative coordinates $\delta \mathbf{q}_{j}$, which is defined for each type of joint such that $\delta \pi_{i j}=\mathbf{H}_{j}\left(\mathbf{A}_{i}, \mathbf{q}_{j}\right) \delta \mathbf{q}_{j}$. The virtual rotation is expressed by $\delta \boldsymbol{\pi}_{j}=\delta \boldsymbol{\pi}_{i}+\delta \xi_{i j}+\mathbf{H}_{j} \delta \mathbf{q}_{j}$.

Equation (19) and the virtual rotation are combined in matrix form as

$$
\begin{gathered}
{\left[\begin{array}{c}
\delta \mathbf{r}_{\mathrm{j}}+\widetilde{\mathbf{r}}_{\mathrm{j}} \delta \boldsymbol{\pi}_{\mathrm{j}} \\
\delta \boldsymbol{\pi}_{\mathrm{j}}
\end{array}\right]=\left[\begin{array}{c}
\delta \mathbf{r}_{\mathrm{i}}+\widetilde{\mathbf{r}}_{\mathrm{i}} \delta \boldsymbol{\pi}_{\mathrm{i}} \\
\delta \boldsymbol{\pi}_{\mathrm{i}}
\end{array}\right]+\left[\begin{array}{c}
\mathbf{A}_{\mathrm{i}} \\
\mathbf{0}
\end{array}\right] \delta \mathbf{s}_{\mathrm{ij}}^{\prime}+} \\
{\left[\begin{array}{c}
\frac{\partial \mathbf{d}_{\mathrm{ij}}}{\partial \mathbf{q}_{\mathrm{j}}}+\widetilde{\mathbf{r}}_{\mathrm{j}} \mathbf{H}_{\mathrm{j}} \\
\mathbf{H}_{\mathrm{j}}
\end{array}\right] \delta \mathbf{q}_{\mathrm{j}}+\left[\begin{array}{c}
\widetilde{\mathbf{r}}_{\mathrm{j}}-\widetilde{\mathbf{d}}_{\mathrm{ij}} \\
\mathbf{I}
\end{array}\right] \delta \xi_{\mathrm{ij}}}
\end{gathered}
$$

In state-vector notation, Eq. (20) is written as

$$
\delta \hat{\mathbf{z}}_{j}=\delta \hat{\mathbf{z}}_{i}+\mathbf{B}_{j} \delta \mathbf{q}_{j}+\mathbf{M}_{i} \delta \mathbf{s}_{i j}^{\prime}+\mathbf{N}_{j} \delta \xi_{i j}^{\prime}
$$

where $\mathbf{M}_{i}=\left[\begin{array}{ll}\mathbf{A}_{i} & \mathbf{0}\end{array}\right]^{T}$ and $\quad \mathbf{N}_{j}=\left[\begin{array}{ll}\widetilde{\mathbf{r}}_{j}-\tilde{\mathbf{d}}_{i j} & \mathbf{I}\end{array}\right]^{T}$

This state representation characterized by Eq. (21) will be used in obtaining a linear set of equations that can automatically be solved for an appropriate configuration.

\section{COMPUTING A SOLUTION FROM A NON-SQUARE JACOBIAN}

The general case of the variation of the constraint $\Phi$ in linearized form can be written as

$$
\delta \Phi=\Phi_{\hat{\mathbf{z}}_{j}} \mathbf{B} \delta \mathbf{q}_{j}+\Phi_{\hat{\mathbf{z}}_{j}} \mathbf{M}_{i} \delta \mathbf{s}_{i j}^{\prime}+\Phi_{\hat{\mathbf{z}}_{j}} \mathbf{N}_{j} \delta \xi_{i j}+\Phi_{\mathbf{s}_{i j}} \delta \mathbf{s}_{i j}+\Phi_{\xi_{i j}} \delta \xi_{i j}
$$

or simply represented by

$$
\Phi_{\mathbf{q}} \delta \mathbf{q}=-\Phi
$$

where $\mathbf{q}$ is the vector of generalized coordinates. When a given initial estimate does not satisfy the constraint equations $\Phi$, the linearized variational constraints are simultaneously solved to obtain an admissible solution. Note that the number of variables is more than the number of constraints. Since the Jacobian of $\Phi(\mathbf{q})$ is not square, the problem of obtaining an assembled configuration can be solved using the MoorePenrose pseudo inverse [9]. Starting with an initial guess $\mathbf{q}^{1}$, the new generalized coordinates are calculated by evaluating

$\Delta \mathbf{q}=\Phi_{\mathbf{q}}^{*}(-\Phi)$

where $\Phi_{\mathbf{q}}^{*}$ is the Moore-Penrose pseudo inverse of the Jacobian $\Phi_{\mathbf{q}}=\left[\partial \Phi_{i} / \partial q_{j}\right]$, defined by

$\Phi_{\mathbf{q}}^{*}=\Phi_{\mathbf{q}}^{T}\left(\Phi_{\mathbf{q}} \Phi_{\mathbf{q}}^{T}\right)^{-1}$

The new set of generalized coordinates is iteratively computed as $\mathbf{q}^{i+1}=\mathbf{q}^{i}+\Delta \mathbf{q}^{i}$ until the constraint function $\|\Phi\|<\mathcal{E}$ is satisfied. The method converges to a solution $\mathbf{q}^{*}$ within a few iterations because of its quadratic rate of convergence. This method will yield a solution set $\mathbf{q}$ that is closest to the original configuration but with changed dimesnions. The final solution characterizes an assembled mechanism that satisfies all kinematic relations imposed by the designer. This new 
mechanism can now be converted back to a mechanical part. The overall method is illustrated in Fig. 4.

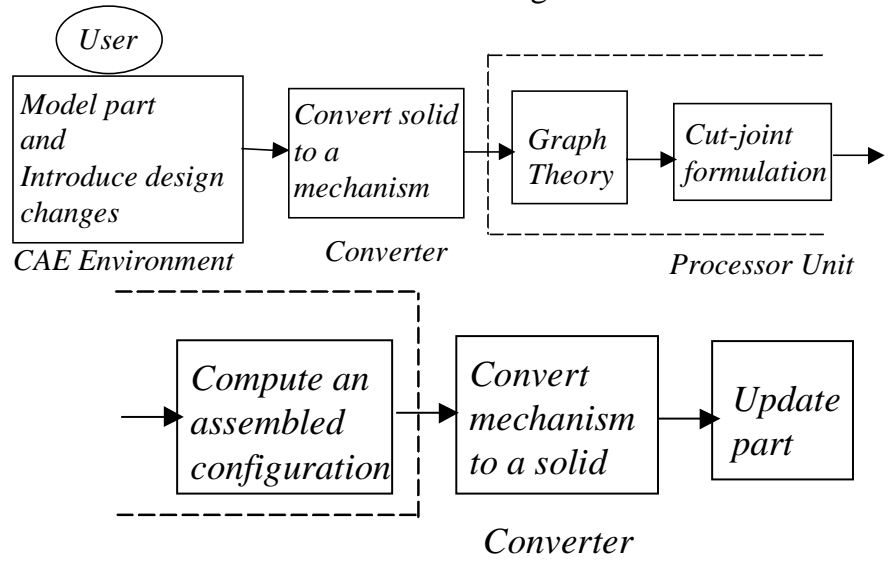

Fig. 4 Block diagram of the overall methodology

EXAMPLE I

Consider the mechanical part shown in Fig. 5a and its cross section shown in Fig. 5b. Due to mating part requirements, an increase in the slot radius $r$ to $r+d r$ is introduced. It required, however, to maintain the distance $d_{1}$ as constant due to assembly requirements but changes in the dimension $d_{2}$ and the angle $\beta$ of the slot are allowed. Although this is a $3 \mathrm{D}$ part, the resulting problem is planar as the mechanism is planar.

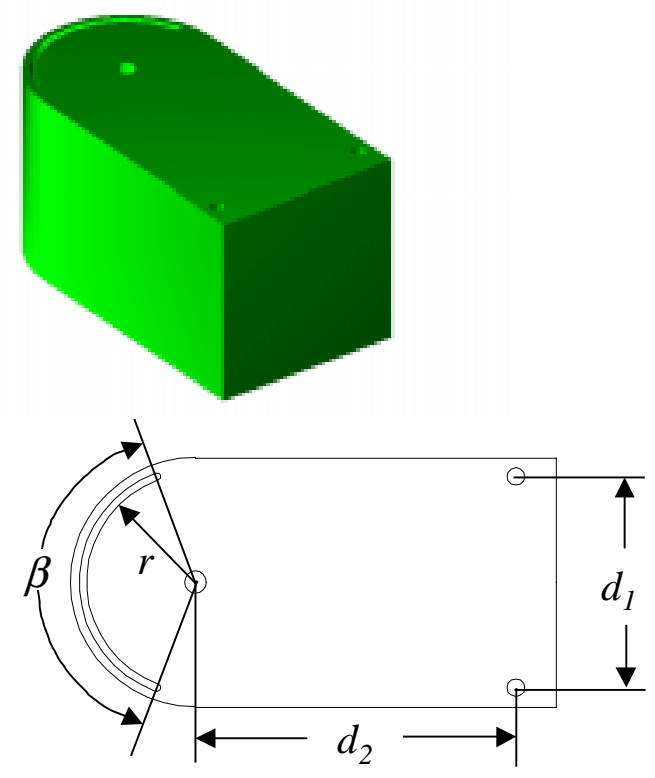

Fig. 5 (a) A mechanical part and its cross section (b) A cross section of the part

To kinematically model this mechanical part, five revolute joints $J_{1}, J_{2}, J_{3}, J_{5}$, and $J_{6}$, and two prismatic joints $J_{4}$ and $J_{7}$ are introduced as shown in Fig. 6. The prismatic joint mandates a horizontal movement (change) of the dimension $d_{2}$ while maintaining the dimension $d_{1}$. The revolute joint at $J_{1}$ is fixed to the ground (i.e., does not translate). Links $0,1,2,3,4$, and 5 are introduced and the connectivity is shown where Link 0 is ground.

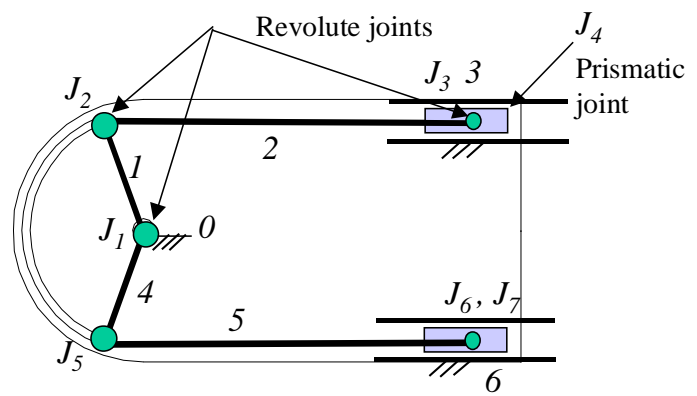

Fig. 6. Kinematic modeling of the mechanical part body the paper here.

Due a design change, a variation is to occur in the Euler vector $\mathbf{p}_{01}$ associated with the transformation matrix $\mathbf{C}_{01}$. To form a spanning tree, the joint connecting links 1 and 2 and that connecting link 4 and 5 are cut, where the bodies do not remain connected after cutting the joint. Note that both sides are symmetric and one computational analysis is enough. Since a joint was cut, a constraint function is introduced such that

$$
\Phi^{r}=\mathbf{r}_{2}+\mathbf{s}_{21}-\mathbf{s}_{12}-\mathbf{r}_{1}=\mathbf{0}
$$

where $\mathbf{S}_{12}$ is the joint-position vector in the reference frame of link 1; and $\mathbf{S}_{21}$ is the joint-position vector in the reference frame of link 2. The Jacobian matrix of the cut-joint in the reference frame of link 1, written in Cartesian coordinate space is

$$
\Phi_{\hat{\mathbf{z}}_{1}}^{r}=\left[\begin{array}{ll}
-\mathbf{I} & \widetilde{\mathbf{r}}_{1}+\widetilde{\mathbf{s}}_{12}
\end{array}\right]
$$

In order to transform the Jacobian matrix from Cartesian coordinate space to joint coordinate space, the velocity transformation matrices are used such that

$$
\Phi_{\mathbf{q}}^{r}=\left[\begin{array}{lll}
\Phi_{\mathbf{z}_{1}}^{r} \mathbf{B}_{\text {rev }} & \Phi_{\hat{\mathbf{z}}_{2}}^{r} \mathbf{B}_{\text {slid }} & \Phi_{\hat{\mathbf{z}}_{2}}^{r} \mathbf{B}_{\text {rev }}
\end{array}\right]
$$

where the velocity transformation matrix of the revolute joint is $\quad \mathbf{B}_{\mathrm{rev}}=\left[\begin{array}{ll}\mathbf{\mathbf { r }}_{1} \mathbf{h}_{01} & \mathbf{h}_{01}\end{array}\right]^{T}, \quad$ and the vector $\mathbf{h}_{01}=\mathbf{A}_{0} \mathbf{C}_{01}\left[\begin{array}{lll}0 & 0 & 1\end{array}\right]^{T}$ is along $\mathbf{z}_{01}^{\prime \prime}$ in the global reference frame.

Using the linearized equations of Section 4, the iterative algorithm is employed until constraint violations are satisfied, where $\mathbf{p}_{03}$ is the vector of Euler parameters associated with $\mathbf{C}_{03}$ (transformation matrix from translational joint coordinates to ground reference frame); $\mathbf{p}_{32}$ is the vector of 
Euler parameters associated with $\mathbf{C}_{32}$. The computed configuration of the mechanism is shown in Fig. 7a, the original part in $7 \mathrm{~b}$, and the updated part in $7 \mathrm{c}$.

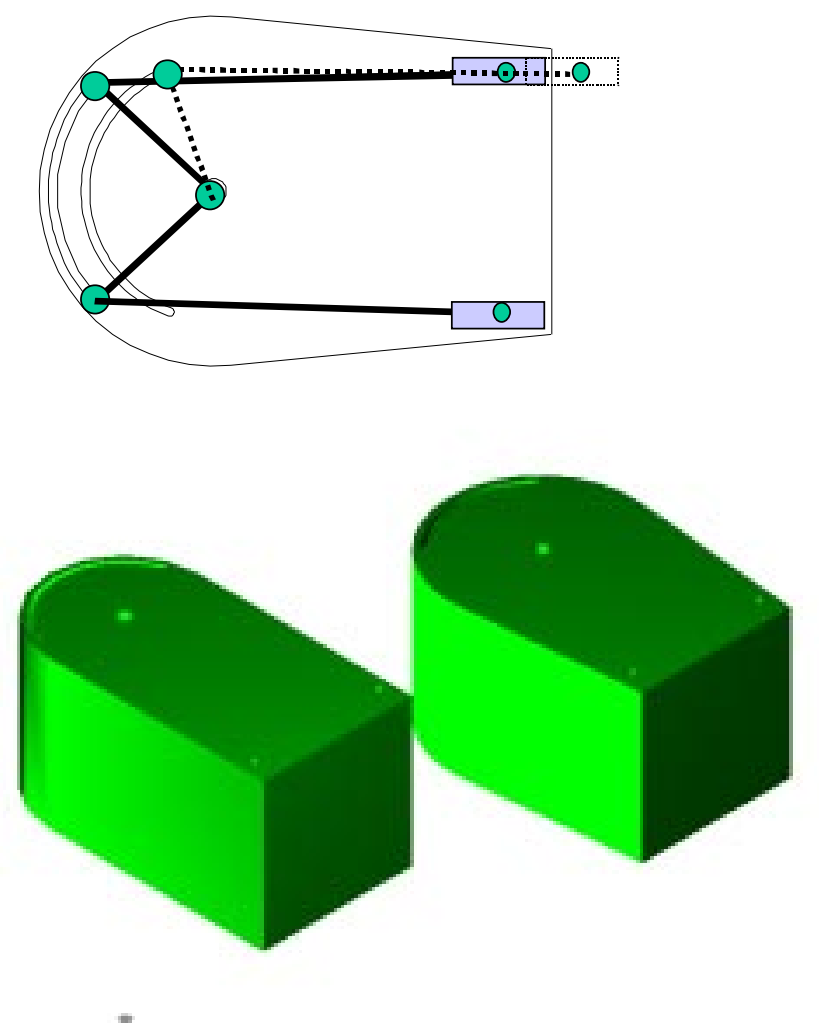

Fig. 7a. Computed change in the mechanism (b) Original part (c) Updated part

\section{EXAMPLE II}

Consider the mechanical part shown in Fig. 8a. In order to compensate for a change in the dimensions of a mating part (not shown), the dimension $n$ is required to change to $n+\partial n$. However, a constraint is introduced such that the distance $d$ in Fig. 8a is unchanged.

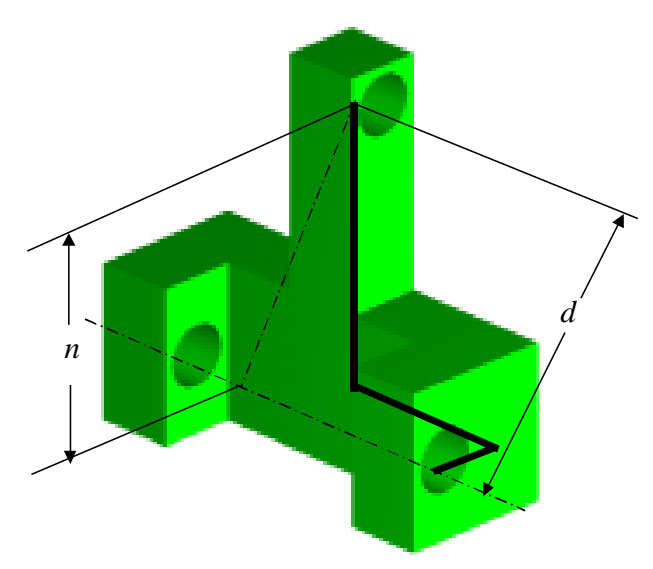

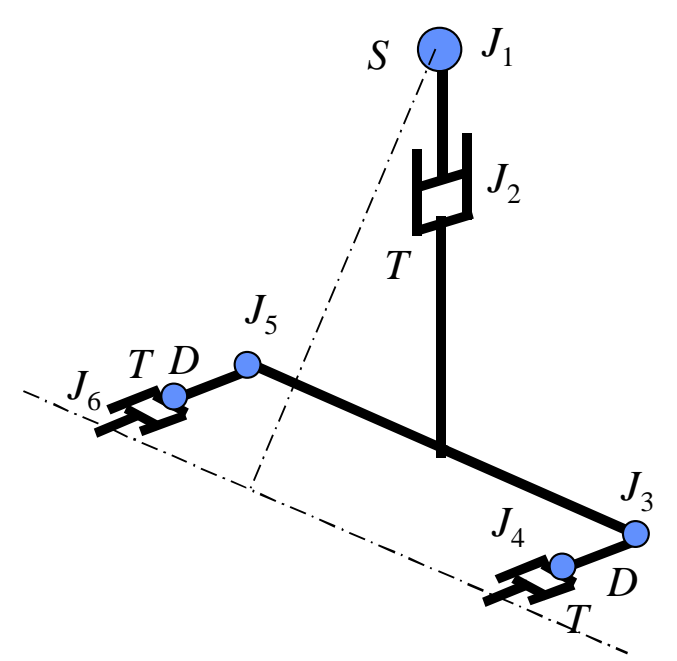

Fig. 8 (a) Mechanical part (b) Kinematic model of the part

The designer selects the type of joints and their locations. Joints $J_{2}, J_{4}$, and $J_{6}$ are translational joints, $J_{3}$ and $J_{5}$ are distance joints, and $J_{1}$ is a spherical joint. The distance joint followed by a translational joint, in this case, specifies a minimum distance that must be maintained but also allows for deformation in the part allowing the distance to be kept.

The system is carefully chosen to allow for distortions in certain geometric parameters. The model comprises two composite Translational-Distance joints $(T-D)$ and one composite Spherical-Translational $(S-T)$ joint as shown in Fig. $8 b$.

To form a spanning tree structure, the two composite $T-D$ joints are cut. The graph-tree representation of the system after cutting joints is shown in Fig. 9b.
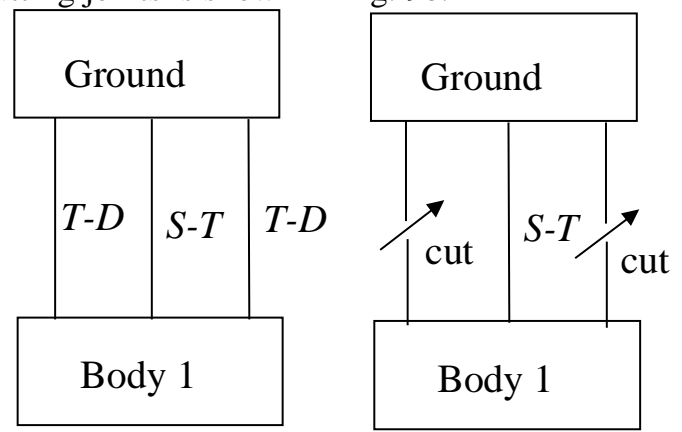

Fig. 9 (a) Graph representation of the kinematic model (b) Cutting joints

Since the $S$ - $T$ joint connects the two entities (ground and body) with a spherical joint and a translational joint, define the vector $\mathbf{S}_{12 S-T}$ as the $S-T$ joint-attachment vector in ground reference frame, and let $\ell_{\mathrm{T}-\mathrm{D}}$ be the constraint distance. Using the proposed formulation, the linear systems of equations in terms of the Jacobians for this mechanism are written as 


$$
\left[\begin{array}{cc}
\Phi_{\mathbf{q}_{S-T}^{S-T}}^{S} & \mathbf{0} \\
\Phi_{\mathbf{q}_{T-D}^{T-D}}^{T} & \Phi_{q_{3}}^{T-D}
\end{array}\right]\left[\begin{array}{c}
\delta \mathbf{q}_{S-T} \\
\delta q_{3}
\end{array}\right]=\left[\begin{array}{c}
-\Phi^{S-T} \\
-\Phi^{T-D}
\end{array}\right]
$$

where $\Phi_{\mathbf{q}_{S-T}}^{S-T}$ and $\Phi_{\mathbf{q}_{S-T}}^{T-D}$ are the Jacobian matrices in joint coordinate space, and are transformed from the Cartesian space $\Phi_{\hat{\mathbf{z}}_{j}}$ by using the velocity transformation matrix $\mathbf{B}$, such that $\Phi_{\mathbf{q}_{S-T}}^{S-T}=\Phi_{\hat{\mathbf{z}}_{j}}^{S-T} \mathbf{B}_{j}^{S-T}$ and $\Phi_{\mathbf{q}_{S-T}}^{T-D}=\Phi_{\hat{\mathbf{z}}_{j}}^{T-D} \mathbf{B}_{j}^{T-D}$.

Estimates of transformation matrices $\mathbf{C}_{i j}$ from Joint coordinate to body reference frame are computed as

$$
\begin{aligned}
\mathbf{C}_{12 S-T} & =\left[\begin{array}{ccc}
0 & -1 & 0 \\
1 & 0 & 0 \\
0 & 0 & 1
\end{array}\right] \\
\mathbf{C}_{21 T-D} & =\left[\begin{array}{ccc}
0 & -1 & 0 \\
1 & 0 & 0 \\
0 & 0 & 1
\end{array}\right]
\end{aligned}
$$

and corresponding Euler parameters $\mathbf{P}_{i j}$ are computed as $\mathbf{P}_{12 S-\mathrm{T}}=\left[\begin{array}{lllll}0.707107 & 0 & 0 & 0.707107\end{array}\right]^{T}$

$\mathbf{P}_{I 2 T-D}=\left[\begin{array}{llll}0.5 & 0.5 & 0.5 & 0.5\end{array}\right]^{\mathrm{T}}, \quad$ and initial generalized coordinates are $\quad \mathbf{q}_{1}=\left[\begin{array}{llll}1 / \sqrt{2} & 0 & 0 & -1 / \sqrt{2}\end{array}\right]^{T}$, $q_{2}=-0.425$, and $q_{3}=-0.305$. The linearized equation for allowing joint variable to automatically be computed must be determined. For a given design variation, the generalized coordinates $\mathbf{q}$ are allowed to vary and the resulting linear system of equations is

$$
\Phi_{\hat{\mathbf{z}}_{2}} \mathbf{B}_{2}^{S-T} \delta \mathbf{q}_{S-T}+\Phi_{q_{3}} \delta q_{3}=-\Phi
$$

Equation (31) is solved for the joint variables using the iterative algorithm. This method yields a new configuration of the mechanism with only varying the generalized coordinates. This is the most direct method and yields results that are easily perceived by the designer prior to modeling. The part is updated accordingly.

Keeping the joint attachment vectors $\mathbf{S}_{i j}$ and transformation matrices $\mathbf{C}_{i j}$ constant, the Newton-Raphson iteration method is used to update the generalized coordinates $\mathbf{q}$.

Upon obtaining an assembled configuration (an admissible solution that satisfies the constraints), a second converter must be used to convert the mechanism to an updated mechanical part. For example, an admissible solution is shown in Fig. 10a and its equivalent mechanical part in Fig. 10b. Simulation results due to a change in $\mathbf{S}_{22 S-T}$ is $\mathbf{q}_{1}=\left[\begin{array}{llll}0.698 & -0.032 & -0.053 & -0.713\end{array}\right]^{T}, q_{2}=-0.434$,

$$
\begin{aligned}
& q_{3}=0.276, \quad \mathbf{S}_{12 S-T}=\left[\begin{array}{lll}
0.501 & 1.116 & 0.199
\end{array}\right]^{T}, \\
& \mathbf{S}_{21 T-D}=\left[\begin{array}{lll}
0 & 1.267 & -0.375
\end{array}\right]^{T}, \\
& \mathbf{S}_{21 T-D}=\left[\begin{array}{llll}
0.07 & 0.155 & -0.186
\end{array}\right]^{T}, \\
& \mathbf{P}_{12 S-T}=\left[\begin{array}{llll}
0.707 & 0 & 0 & 0.707
\end{array}\right]^{T}, \\
& \mathbf{P}_{12 T-D}=\left[\begin{array}{llll}
0.5 & 0 & 0 & 0.5
\end{array}\right]^{T} . \text { The constraint tolerance yielded } \\
& \Phi_{\text {norm }}=0.0006725 \text { after } 2 \text { iterations. Allowing only joint } \\
& \text { variables } \quad \text { to } \quad \text { be } \quad \text { automatically } \quad \text { computed } \quad \text { yielded } \\
& \mathbf{q}_{1}=\left[\begin{array}{llll}
0.698 & 0.0784 & 0.0912 & -0.7166
\end{array}\right]^{T} \\
& q_{2}=-0.3683, \quad q_{3}=0.2349, \quad \mathbf{S}_{12 S-T}=\left[\begin{array}{lll}
0.5 & 1.0 & 0.374
\end{array}\right]^{T}, \\
& \text { after } \Phi_{\text {norm }}=0.000158 \text { after 3 iterations. }
\end{aligned}
$$
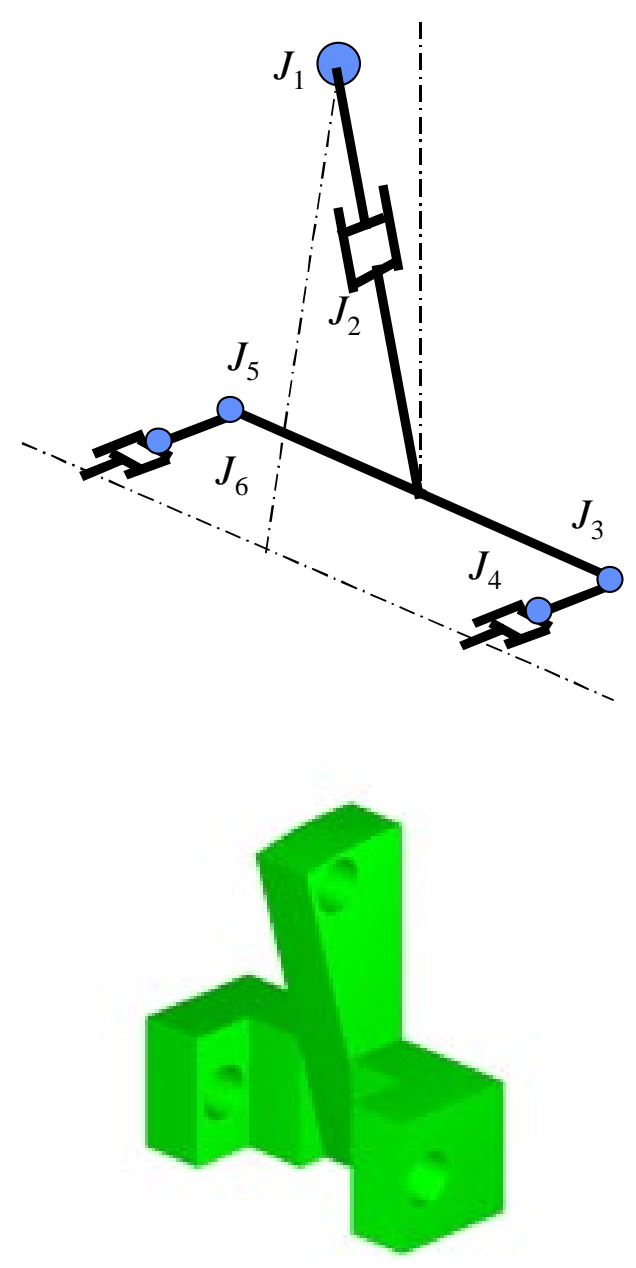

Fig. 10 (a) The mechanism after computing an assembled configuration (b) The equivalent redesigned part 


\section{CONCLUSIONS}

A broadly applicable formulation for automating the redesign of mechanical parts in a concurrent engineering environment was presented. The iterative process typically associated with mechanical design is reduced by the use of such automated methods. Mathematical formulations based on earlier work are further expanded in this paper and are demonstrated to the automated computer-aided design of mechanical parts in a CAD environment. The significance of this formulation is evidenced in that it characterizes an alternative efficient method to the common parametric methods currently used.

It was shown that mechanical parts represented in a CAD environment could be modeled into a mechanism comprising joints and links. It was shown that the deformation of the solid sustained by introducing a design variation is computed using a kinematic model. It was also shown that design changes through the mechanism are propagated such that an admissible configuration is computed using an efficient iterative generalized inverse method. While the experimental computer code used to implement the algorithm is not refined, it has nevertheless demonstrated a new type of automatic design well suited for implementation in concurrent engineering.

\section{ACKNOWLEDGMENTS}

This research was funded by the United States Army Tank-Automotive and Armaments Command (TARDEC) under the Automotive Research Center contract number DAAE-07-94-C-R094.

\section{REFERENCES}

1. Imamura, S, 1994, 'Development of constraint based object oriented language for engineering design-on the parametric design support' Journal of the Japan Society for Precision Engineering Vol 60 No 9, pp1242-1246

2. Burke, E K, Elliman, D G, and Heard, M I, 1994, 'XCODAMS: an engineering design system based on constraint propagation' Applications of Artificial Intelligence in Engineering, pp601-608

3. Cutkosky, M R and Tenenbaum, J M, 1990, "Toward a computational framework for concurrent engineering" Electronics Society-IECON, Pacific Grove, CA, pp700706

4. McMahon, C.A.; Cox, D.R.; Sims Williams, J.H.; Scott, J.A., 1997, "Representation and Reasoning in Computer Aided Process Planning," Proceedings of the Institution of Mechanical Engineers, Part B: Journal of Engineering Manufacture v 211 n B6, MEP London Engl p 473-485.

5. Abdel-Malek, K. and Maropis, N., 1998 "Design-toManufacture Case Study: Automatic Design of PostFabrication Mechanisms for Tubular Components," SME Journal of Manufacturing Systems, Vol. 17, No. 3, pp. 183-195.
6. Zou, H. L., Abdel-Malek, K., and Wang, J. Y., 1996. Computer-Aided-Design Using the Method of Cut-Joint Kinematic Constraints, Computer-Aided Design, Vol 28, No. 10, pp. 795-806.

7. Haug, E. J. Computer Aided Kinematics and Dynamics of Mechanical Systems, Vol I: Basic Methods, Allyn \& Bacon, Boston (1989)

8. Zou, H.L. Abdel-Malek, K. and Wang, J., 1997, Design Propagation in Mechanical Systems: Kinematic Analysis, ASME Journal of Mechanical Design, Vol. 119, No. 3, pp. 338-345.

9. Abdel-Malek, K. and Yeh, H. J., 1997, "On the Determination of Starting Points for Parametric Surface Intersections," Computer-Aided Design, Vol. 29, No. 1, pp.21-35. 